\title{
Síndrome de Erasmus: a propósito de un caso
}

\author{
Julia Romero', María Eugenia Lara1, Eugenia Bacigalupo², Julio Venditti², Martín Bossio³, Mariano Rivero", \\ Silvia Quadrelli ${ }^{3}$, Juan Carlos Barreira ${ }^{1}$ \\ ${ }^{1}$ Servicio de Reumatología, ${ }^{2}$ Servicio de Anatomía Patológica, ${ }^{3}$ Servicio de Neumonología, Hospital Británico de Buenos Aires.
}

\section{Resumen}

En 1957, L. D. Erasmus comunicó la asociación entre sílice y esclerosis sistémica, destacando la importancia de la exposición a sílice como un factor de riesgo para el desarrollo de esclerodermia ocupacional. Si bien existen reportes de la interacción entre sílice y el sistema inmune, continúa siendo actualmente una asociación infrecuente.

El objetivo es presentar un paciente varón de 41 años con esclerosis sistémica e historia de exposición a gran cantidad de polvo de sílice, que desarrolló síndrome de Erasmus. Realizamos además una revisión de la literatura.

\section{Introducción}

La esclerosis sistémica (ES) es una enfermedad autoinmune que se caracteriza por fibrosis tisular, presencia de autoanticuerpos y lesión vascular. Su etiología es desconocida e intervendrían en su patogenia factores genéticos, ambientales y ocupacionales como la exposición a sílice ${ }^{1}$.

La silicosis constituye una enfermedad crónica, fibrogénica, de evolución lenta e irreversible causada por la inhalación de polvo de sílice cristalizado. Las actividades con mayor riesgo son: el tamizado de arena, la minería, el trabajo en canteras, fundiciones, fábrica de cerámicas y piedras ${ }^{2}$.

Es nuestro objetivo comunicar un paciente con ES que desarrolló compromiso pulmonar por sílice durante la evolución de su enfermedad, conociéndose la misma como Síndrome de Erasmus.

\section{Caso clínico}

Varón, de raza blanca, con antecedentes de hipertensión arterial en tratamiento con enalapril $5 \mathrm{mg} /$ día y dislipemia que trabajaba en la industria arenera desde 4 años previos al diagnóstico de ES. Comenzó a los 36 años (2007) con

\section{Summary}

In 1957, L. D. Erasmus reported the association between silica and systemic sclerosis, highlighting the importance of the silica exposure as a risk factor for developing scleroderma occupational. While there are reports of the interaction between silica and the immune system currently remains an infrequent association.

The objective is to report a 41 year old male patient with systemic sclerosis and history of high quantity of silica dust exposure who developed an Erasmus syndrome. A review of the literature has also been performed.

fenómeno de Raynaud, esclerodactilia e induración cutánea proximal, úlceras digitales y en miembros superiores, frotes tendinosos, artritis de pequeñas y posteriormente de grandes articulaciones, y mialgias progresivas sin elevación de enzimas musculares y sin patrón miopático en el electromiograma. A nivel respiratorio presentó disnea clase funcional II y rales "velcro" bibasales. Las pruebas de función respiratoria evidenciaron un patrón restrictivo leve, con opacidad reticulonodulillar difusa bilateral a predominio bibasal en la radiografía de tórax. La tomografía de alta resolución (TACAR) de tórax mostró hallazgos compatibles con neumonía intersticial no específica (NSIP) (Figura 1). Recibió ciclofosfamida $1 \mathrm{mg} /$ $\mathrm{kg} /$ día vía oral durante 6 meses y meprednisona $20 \mathrm{mg} /$ día, concomitantemente con d-penicilamina $250 \mathrm{mg} /$ día, bosentan $250 \mathrm{mg} /$ día, sidenafil $150 \mathrm{mg} /$ día y ácido acetil salicílico $81 \mathrm{mg} /$ día, para el compromiso cutáneo y vascular (úlceras digitales). Presentó progresivo descenso de peso y en su evaluación se halló esofagitis grado A, aperistalsis del cuerpo esofágico e hipotonía del esfínter esofágico inferior, hernia hiatal, gastropatía erosiva y reflujo ácido nocturno, inició tratamiento con esomeprazol $80 \mathrm{mg} /$ día y domperidona $40 \mathrm{mg} /$ día. Posteriormente requirió suplementos nutricionales por síndrome de ma-

\section{Correspondencia}

Julia Romero

E-mail:drajuliaromero@gmail.com 


\begin{tabular}{|l|c|c|c|}
\hline & $\mathbf{2 0 0 7}$ & $\mathbf{2 0 0 9}$ & $\mathbf{2 0 1 1}$ \\
\hline Patrón restrictivo & Leve & Leve & Severo \\
\hline CVF (\%) & 72 & 63 & 40 \\
\hline VEF $_{1} /$ CVF (\%) & 88 & 88 & 43 \\
\hline CPT (\%) & 72 & 72 & 72 \\
\hline Pimáx (\%) & -- & & 89 \\
\hline Pemáx (\%) & -- & & 44 \\
\hline DLCO (\%) & 69 & 59 & 55 \\
\hline $\begin{array}{l}\text { CVF: Capacidad Vital Forzada. VEF1: Volumen Espiratorio Forzado en el primer segundo. } \\
\text { VEF1/CVF: Índice de Tiffeneau. Pimáx.: Presión Inspiratoria máxima. } \\
\text { Pemáx.: Presión Espiratoria máxima. DLC0: Difusión Pulmonar de Monóxido de Carbono. }\end{array}$ \\
\hline
\end{tabular}

Tabla 1. Pruebas de función pulmonar - Evolución.

labsorción. Se detectó: ANA patrón homogéneo en bajos títulos (1/80), anti-Scl70 positivo e hipergammaglobulinemia. La capilaroscopia presentó una patente tardía de esclerodermia (Figura 2).

En septiembre de 2009, por progresión de la disnea con caída de la DLCO del 10\% (Tabla 1) y progresión del daño estructural de la NSIP (Figura 3), recibió 6 pulsos de 1 gramo mensual de ciclofosfamida y meprednisona $10 \mathrm{mg} /$ día, manteniéndose estable la función pulmonar durante 1 año. En 2011 empeoró a clase funcional III, con patrón restrictivo severo y descenso de DLCO a 55\% (Tabla 1), hallando en la TACAR múltiples signos parenquimatosos y mediastínicos que sugerían enfermedad granulomatosa asociada (Figura 4). La presión de la arteria pulmonar medida por ecodoppler fue de $50 \mathrm{mmHg}$, por lo que se agregó al tratamiento previo, diltiazem $180 \mathrm{mg} /$ día.

Por la falta de respuesta clínica al tratamiento y deterioro de la función respiratoria a los dos meses de iniciado el mismo, se realizó biopsia pulmonar por videotoracoscopia, hallando una marcada distorsión de la histoarquitectura que adoptaba un aspecto "panalizado" por engrosamiento fibroso septal, focos fibroblásticos y proliferación de bandas de músculo liso. Coexistía hiperplasia de neumocitos tipo II y moderado infiltrado inflamatorio, de predominio linfocitario, compatible con fondo de neumonía intersticial usual (UIP) y numerosos nódulos tipo silicóticos predominantemente subpleurales (Figuras 5 y 6). Completó segundo ciclo de 6 pulsos de 1 gramo de ciclofosfamida sin progresión clínica ni tomográfica.

En 2012 presentó cambios en electrocardiograma, con bloqueo trifascicular y extrasístoles ventriculares aisladas

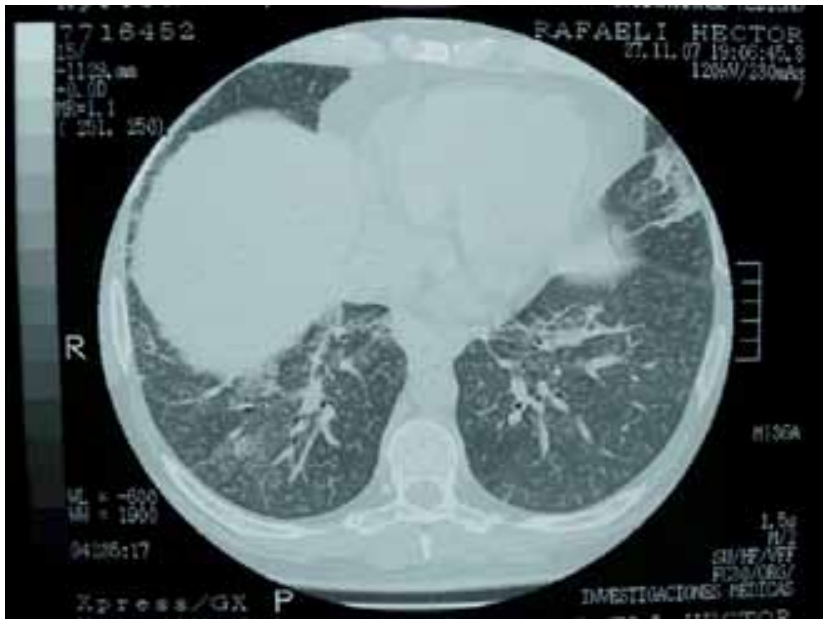

Figura 1. TACAR de tórax de 2007. Intersticiopatía bilateral difusa de tipo reticulonodulillar.

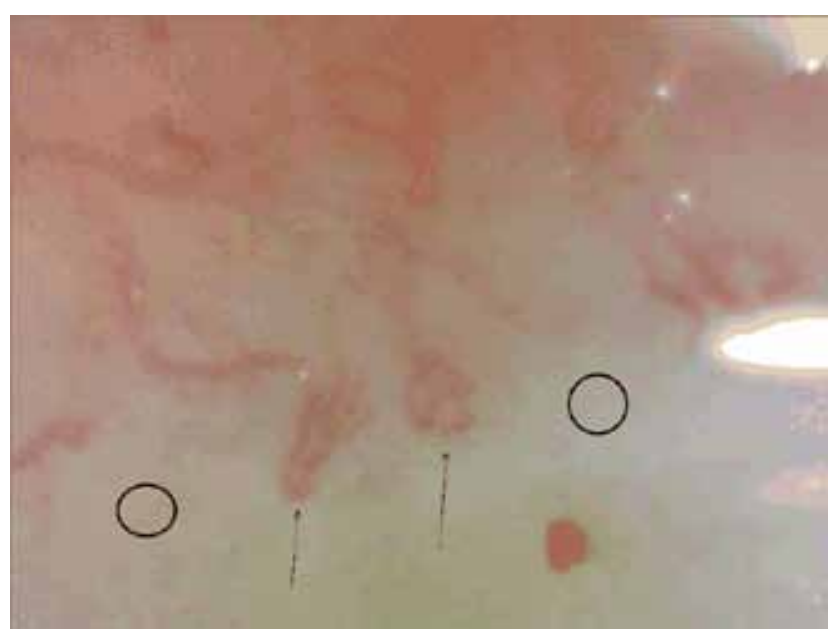

Figura 2. Patrón capilaroscópico de fase tardía. Se observa angiogénesis (flechas) intercalada con áreas netamente avasculares (círculos).

que requirió colocación de marcapaso bicameral. Fallece a los 5 años del diagnóstico por muerte súbita en su domicilio.

\section{Discusión}

En nuestro paciente el diagnóstico de esclerodermia fue previo al de silicosis, llegando al mismo a través de la biopsia, motivada por las imágenes de la TACAR de tórax.

El diagnóstico de ES se basa en criterios clínicos y de laboratorio, de acuerdo al American College of Rheumatology $(\mathrm{ACR})^{3}$. El compromiso pulmonar ocurre en aproximadamente el $70 \%$ de los pacientes, ya sea como afectación intersticial o vascular. Entre las enfermedades 


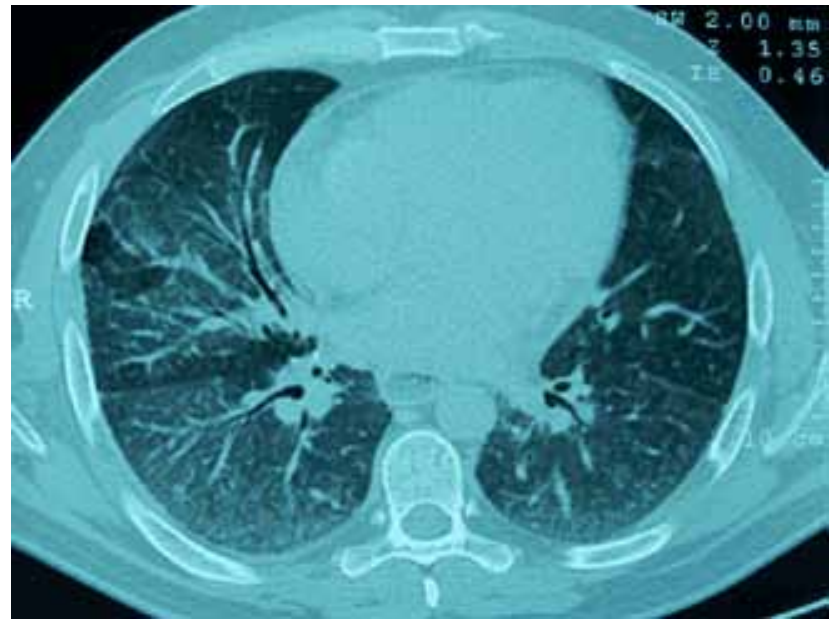

Figura 3. TACAR de 2009. Engrosamiento de septos interlobulillares y áreas en vidrio esmerilado de distribución periférica con bronquio y bronquioloectasias.

intersticiales, en la ES, el patrón de mayor prevalencia es la NSIP 4 .

Nuestro paciente desarrolló ES luego de un corto tiempo de exposición a sílice. A diferencia de otras profesiones, los trabajadores expuestos a grandes cantidades de sílice en el tamizado de arena justificaría el tiempo relativamente corto para el desarrollo de la misma. La presencia de micronódulos difusos y bilaterales, predominantemente en lóbulos superiores y de calcificaciones ganglionares “en cáscara de huevo" observadas en la tomografía son características de silicosis pulmonar ${ }^{5}$.

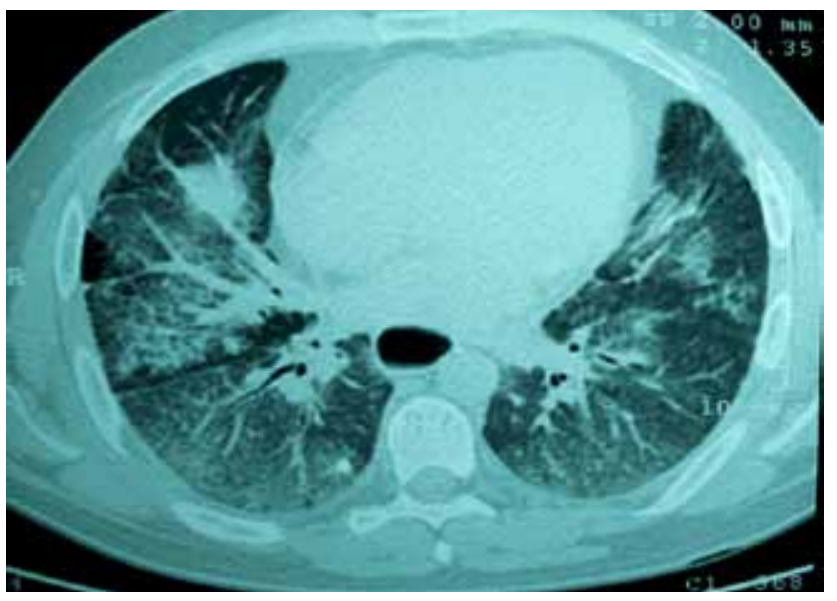

Figura 4. TACAR de 2011. Engrosamiento de septos interlobulillares asociados a áreas en vidrio esmerilado de distribución periférica. Opacidades nodulillares en cisuras "signo de la perla enhebrada". Granuloma calcificado en segmento posterior del lóbulo inferior izquierdo. Múltiples estructuras ganglionares mediastínicas calcificadas.
Las TACAR de tórax de 2007 y 2009 presentan características del compromiso pulmonar en la ES, con predominio de lesiones intersticiales y bronquioloectasias en zonas periféricas y basales de ambos pulmones. Sin embargo, en la tomografía de 2011, la presencia de microcalcificaciones ganglionares mediastínicas y el "signo de la perla enhebrada” hicieron sospechar en esta asociación.

La ES y la exposición ocupacional a sílice fue inicialmente comunicada por Erasmus en $1957^{6}$. Sanchez Roman y cols., en un estudio con 300 trabajadores de una fábrica que utilizaba el tamizado de arena, identificó 50 casos de silicosis, el 10\% de estos asociados a ES'. Castro y cols. hallaron una prevalencia significativa de ES en mineros con silicosis o antracosis, aumentando la posibilidad de que la fibrosis pulmonar podría representar un elemento relevante en la génesis del proceso esclerótico ${ }^{8}$. Un reciente metaanálisis demostró que la exposición a este mineral ocasiona un aumento del riesgo en 3.2 veces para desarrollar ES, mayor en varones?.

Entender los mecanismos por los cuales las partículas de sílice inducen fibrosis pulmonar requiere de más investigaciones. Sin embargo, se sabe que una vez depositado en el tracto respiratorio, estas partículas pueden ser fagocitadas por los macrófagos alveolares o penetrar en el epitelio a través de endocitosis. La ruptura de los fagolisosomas produce la liberación de enzimas y partículas en el medio extracelular, determinando injuria tisular y autolisis. Entre los mediadores liberados por los macró-

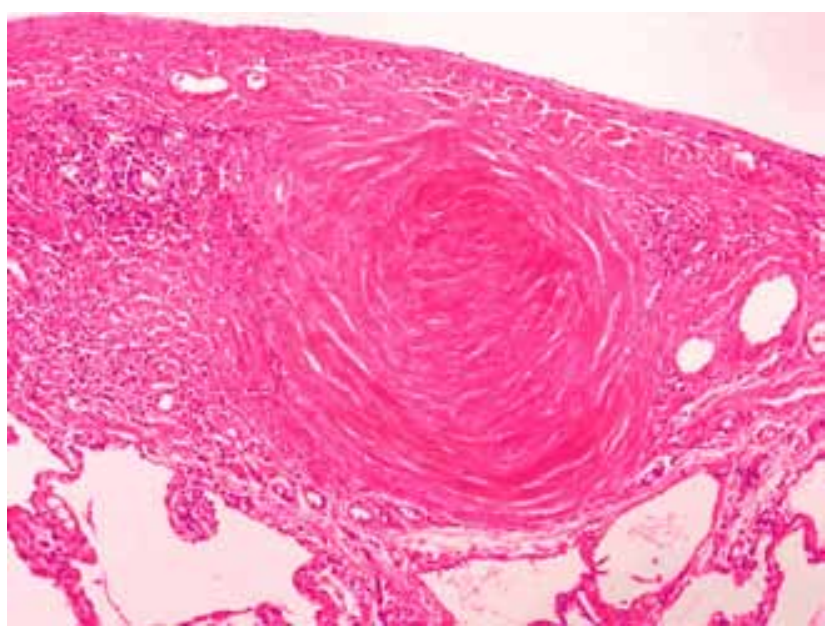

Figura 5. Hematoxilina-Eosina 10X. Lesión subpleural de fibrosis nodular concéntrica (nódulo silicótico) con marcada distorsión de la histoarquitectura pulmonar que adopta un aspecto panalizado con engrosamiento fibroso septal, focos fibroelásticos y proliferación de bandas de músculo liso. 


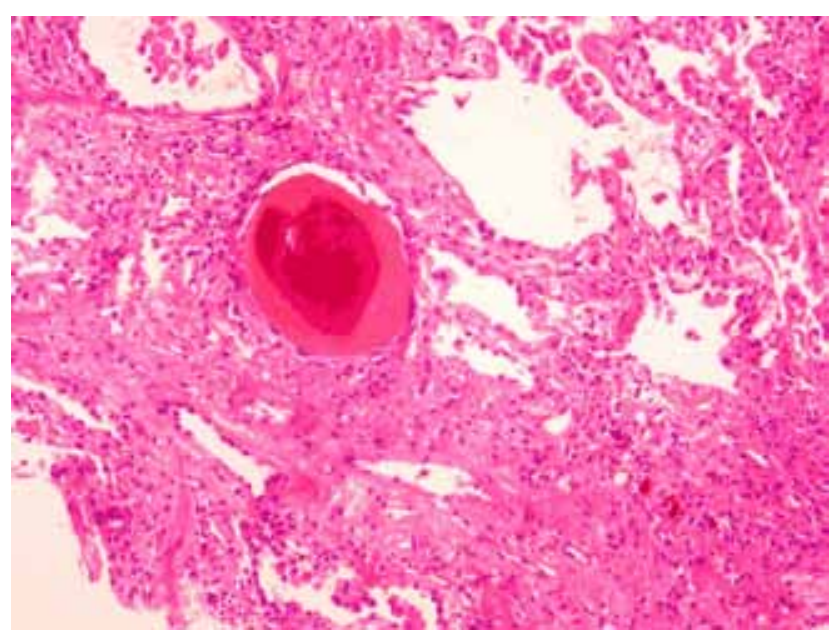

Figura 6. Hematoxilina-Eosina 40X. Nódulo silicótico hialinizado y calcificado. Coexiste hiperplasia de neumocitos tipo II y moderado infiltrado inflamatorio predominantemente linfocitario.

fagos activados, que promueven el crecimiento de los fibroblastos se incluyen: la interleuquina-1 (IL-1), factor de necrosis tumoral alfa (TNF- $\alpha$ ), factor de crecimiento símil insulina (IGF-1), factor de crecimiento derivado de plaquetas (PDGF), factor de crecimiento transformador beta (TGF) y fibronectina. Así, como la silicosis se asocia a menudo con anormalidades humorales y celulares en sangre periférica, con el desarrollo de anticuerpos antinucleares, factor reumatoideo, hipergammaglobulinemia y aumento en el número de linfocitos T helper y supresores $^{10}$. De esta forma, la producción de autoanticuerpos y la formación de complejos inmunes que circulan en relación con la citotoxicidad de sílice, sería el enlace para explicar el desarrollo de enfermedades autoinmunes.

En nuestro medio, la silicosis es considerada dentro de las neumoconiosis causadas por polvo mineral fibrogénico, considerándose un carcinógeno para el hombre pero sin mención de su relación con el desarrollo de enfermedades autoinmunes ${ }^{11}$.

En los pacientes con exposición ocupacional a minerales, que desarrollen enfermedad intersticial y alteraciones inmunológicas, que no respondan al tratamiento habitual debería contemplarse la realización de una biopsia pulmonar que contribuya a establecer las causas asociadas.

\section{Bibliografía}

1. Steen VD. Occupational scleroderma. Curr Opin Rheumatol. 1999 Nov; 11(6):490-4.

2. Souza PFM, Figueiredo RC, Klumb EM, Albuquerque EMN, Lopes AJ, Capone D, Jansen JM. Associação entre silicose e esclerose sistêmica - Síndrome de Erasmus. Pulmão RJ. 2005; 14(1):79-83.

3. Masi AT, Rodnan GP, Medsger TA. Preliminary criteria for the classification of systemic sclerosis (sclerodermia). Subcommittee for scleroderma criteria of the American Rheumatism Association Diagnostic and Therapeutic Criteria Committee. Arthritis Rheum. 1980 May; 23(5):581-90.

4. Stenn VD, Conte C, Owens GR y Medsger TA Jr. Severe restrictive lung disease in systemic sclerosis. Arthritis Rheum. 1994 Sep; 37(9):1283-9.

5. Capone D, Mogami R, Miyagui T. Tomografia computadorizada de alta resolução nas doenças difusas pulmonares - correlação anatomopatológica. São Paulo - Rio de Janeiro - Ribeirão Preto - Belo Horizonte: Atheneu; 2003; 360.

6. Erasmus LD. Scleroderma in gold-miners on the Witwatersrand and with particular reference to pulmonary manifestations. S Afr J Lab Clin Med. 1957 Sep 3(3):209-31.

7. Sanchez Roman J, Wichmann I, Salaberri J, Varela JM, Nuñez Roldan A. Multiple clinical and biological autoimmune manifestations in 50 workers after occupational exposure to silica. Ann Rheum Dis. 1993(7); 52:534-8.

8. Castro HA, Silva CG, Lemle A. Estudo de imunoglobulinas, complementos e autoanticorpos em 58 trabalhadores expostos à sílica. J Bras Pneumol 2004; 30(3):201-6.

9. McCormic ZD, Khuder SS, Aryal BK, Ames AL, Khuder SA. Occupational silica exposure as a risk factor for scleroderma. Int Arch Occup Environ Health. 2010 Oct; 83(7):763-9.

10. Mora GF. Systemic Sclerosis: Environmental Factors.J Rheumatol. 2009 Nov; 36(11):2383-96.

11. Albiano NF. Toxicología laboral. Criterios para el monitoreo de la salud de los trabajadores expuestos a sustancias químicas peligrosas. Superintendencia de riesgos del trabajo. 2011. Cap. 10. 247-50. 